\title{
Teaching Physical Collaborative Tasks: Object-Lifting Case Study with a Humanoid
}

\author{
Paul Evrard, Elena Gribovskaya, Sylvain Calinon, Aude Billard and Abderrahmane Kheddar
}

\begin{abstract}
This paper presents the application of a statistical framework that allows to endow a humanoid robot with the ability to perform a collaborative manipulation task with a human operator. We investigate to what extent the dynamics of the motion and the haptic communication process that takes place during physical collaborative tasks can be encapsulated by the probabilistic model. This framework encodes the dataset in a Gaussian Mixture Model, which components represent the local correlations across the variables that characterize the task. A set of demonstrations is performed using a bilateral coupling teleoperation setup; then the statistical model is trained in a pure follower/leader role distribution mode between the human and robot alternatively. The task is reproduced using Gaussian Mixture Regression. We present the probabilistic model and the experimental results obtained on the humanoid platform HRP2; preliminary results assess our theory on switching behavior modes in dyad collaborative tasks: when reproduced with users which were not instructed to behave in either a follower or a leader mode, the robot switched automatically between the learned leader and follower behaviors.
\end{abstract}

\section{INTRODUCTION}

Our aim is to accomplish physical collaborative tasks between a human and a humanoid robot. In robotics, this problem has lately been referred to as physical Human-Robot interactions (pHRI). Physical interaction between a couple of human or robotic agents considers situations where the agents are in physical contact with each other and exchange mechanical energy. The contact can be direct, i.e. part of the human body is in contact with part of the robot links; it can also be established through collaborative manipulation of a common object. Our work concerns the latter case, and aims at endowing a humanoid with collaborative manipulation skills so as to cooperate with a human partner in a proactive way.

To perform pHRI tasks, the partners concurrently apply forces on a common object of interest. During the task, the partners regulate the exchange of mechanical energy not only based on task and environmental physical requirements but also through mutual understanding of each other's intentions in order to negotiate a common trajectory or a strategy which allows performing the task in a good synergy. If we consider that each partner can take initiatives, the adaptation and the

Paul Evrard and Abderrahmane Kheddar are with the Centre National de la Recherche Scientifique (CNRS): CNRS-UM2 LIRMM, Montpellier, France, and the CNRS-AIST JRL, UMI3218/CRT, Tsukuba, Japan. evrard.pauldaist.go.jp, kheddardieee.org

Elena Gribovskaya and Aude Billard are with the Learning Algorithms and Systems Laboratory. elena.gribovskaya@epfl.ch, aude.billardepefl.ch

Sylvain Calinon is with the Italian Institute of Technology. sylvain.calinondit.it synchronization among the partners is certainly bilateral, i.e. both partners adjust and adapt to each other. In the literature, very little is known on these processes in humans, though being able to understand and model them would be a great advance towards robot partners' technology.

The ability to negotiate and perform a collaborative task is acquired by humans at an early age, but this cognitive process is still not well understood and hence it is difficult to infer hypotheses or models that one can be inspired from to design solutions for robotic systems. State-of-the-art systems based on impedance control [1] provides interesting bricks in understanding what could probably be sub-modules of the overall solution. Many implementation show interesting performances [2], [3], [4], [5], [6], yet they affix the robot a pure follower role. Though one may say that, to some extent, a robot can perform simple collaborative tasks, it certainly is unable to negotiate them with their human partners.

We claim that during a dyadic physical collaborative task, both partners regulate the behavior between two extreme theoretical cases: a leader and a follower behaviors [7]; we also claim that the partners rely on hidden force patterns in the haptic signal exchanged while performing the task to relay intention through haptic communication. We also believe that this haptic communication can be used as a modality to adjust a weighting function (a homotopy) between the leader and follower roles [8]. However, we admit that these theoretical hypotheses are yet difficult to assess: the nature of the force patterns and the hypothetical haptic language that is supposed to relay user's intentions are probably appealing theories, but they need to be proved.

Our idea in proving these concepts is to tackle the pHRI problem by using a Programming by Demonstration (PbD) approach, described in [9] and on which this work builds. We hypothesize that part of the complexity of deriving explicit models can be avoided by using a probabilistic framework based on Gaussian Mixture Models (GMM) and Gaussian Mixture Regression (GMR). We also want to investigate whether this framework is able to catch all the aspects of the haptic cue exchange during collaborative physical task, including the dynamics of the motion and the synchronization and adaptation processes.

In [9], a robotic system is demonstrated several instances of a collaborative lifting task, and learns the necessary skills to reproduce the task. The reproduction of the task is then simulated to evaluate the learned model. This paper presents the results from a real experiment involving the HRP-2 humanoid robot jointly lifting an object with a human operator. We will try to use obtained results to see assess our 
behavior switching theory developed in [7], [8].

\section{DEMONSTRATING COLLABORATIVE TASKS}

\section{A. Human-Robot collaborative lifting tasks}

If two partners perform a lifting task collaboratively, they are likely to have different intentions regarding the target position of the lifted object and the desired velocity profile [10]. Hence, during the motion, they will have to adjust toward a common plan. We make the hypothesis that this is partly achieved through haptic cues, i.e. that the partners will guess each other's intentions by interpreting kinesthesia and the interaction forces they sense at the grasping points of the manipulated object. This hypothesis relies on the fact that a disagreement between the partners will result in higher interaction forces.

We hereby consider a one-dimensional task where the position of the lifted object is described by its altitude. Besides, we also consider the vertical velocity of the object and the force applied by the robot along the vertical axis. We assume that the mass of the object is known, and more importantly, that the task will be demonstrated in predetermined stereotypical manners, where one partner is asked to always lead and the other is asked to act as a pure follower at the onset and at the end of the motion. Finally, we also assume that the partner acting as a follower is blindfolded, so that visual cues do not interfere with the task.

Rahman et al. showed that the dynamics of a follower human arm during collaborative task was dominated by a variable damping term [4]. The force applied on the robot's wrist and the gripper velocity should therefore be taken into consideration in order to reproduce human-like dynamics during the task. In our case, we consider that the robot can also have its own desired trajectory to perform the task. A position-dependent term can be added to the dynamics to drive the arm towards a target position.

In the context of this work, we will thus use the following three variables to characterize the behavior of the robot during collaborative lifting tasks: the altitude $x$ of the robot's gripper and its velocity $\dot{x}$, and the force $F$ sensed by the robot at the grasping point.

\section{B. Robotic system and controller}

Collaborative lifting tasks have been demonstrated on the full-sized humanoid robot HRP-2. Fig. 1 shows the experimental setup used during the teaching phase, see [9] for a thorough description of the setup. During the demonstrations, a human operator (the teacher) teleoperates the robot using a 6dof force feedback device described in [11]. A second operator (the user) assists the teleoperated robot to lift a beam, while keeping it horizontal. Only the right arm is used to perform the lifting task, while the robot is standing. The wrist of the robot is constrained to move only along a vertical direction during the whole task, and its orientation is constrained to remain constant.

The force feedback device and the robot's arm are bilaterally coupled using a simple 2-channel Velocity-Force

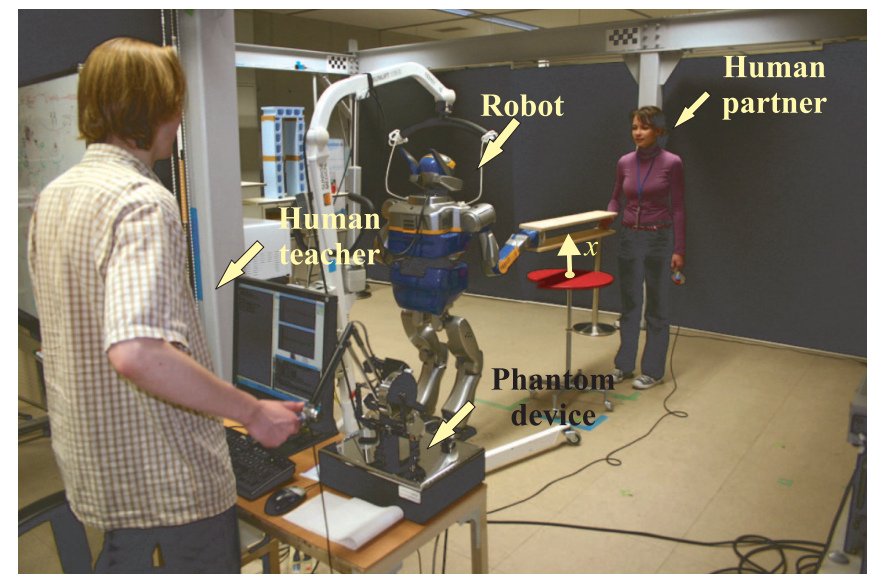

Fig. 1. A human (teacher) teleoperates the humanoid HRP-2 through a force feedback haptic display to teach HRP-2 the lifting task made collaboratively with another human (task-partner).

scheme. The velocity of the tip of the PHANToM device is sent as a velocity reference for the end-effector of the robot. The forces and torques measured at the wrist of the robot were scaled and mapped to the PHANToM workspace to be sent as a reference for the low-level control of the device. The value scale factor applied to the robot sensor forces was set to 0.3 . This scale factor is applied for stability reasons. Another option to ensure the stability of the system is to damp the system. This allows feeding back the sensor's forces with their real amplitude; however it limits the velocity of the motion, resulting in less realistic demonstrations. Both options have been tested, and rescaling the forces has been chosen, as it felt more natural to the teacher.

\section{Method and results}

Two sets of scenarios are demonstrated to the robot. In the first set, the teacher closes the eyes and the user initiates and ends the motion: this is the case where we instructed the robot teacher to behave like a pure follower where the partner is instructed to behave as a pure leader. In the other set, the roles are exchanged, and the teacher leads the onset and end of the motion. The position and velocity of the robot's gripper together with the force measured at the robot's wrist were measured at $200 \mathrm{~Hz}$. The teaching process was conducted with one teacher (obviously, the robot behavior is personalized to this teacher), and only two humans as taskpartners.

The results are illustrated in Fig. 2 showing separate patterns for the two different teaching behaviors (robot leader and robot follower). See details in [9].

\section{PROPOSED APPROACH}

\section{A. Probabilistic encoding and retrieving of the skill}

Once a task has been demonstrated, the robot has to automatically extract the important features of the task in order to reproduce it [12]. For this purpose, we use a probabilistic framework based on GMMs to encode the correlations between the different variables of the task, and GMR [9] to 


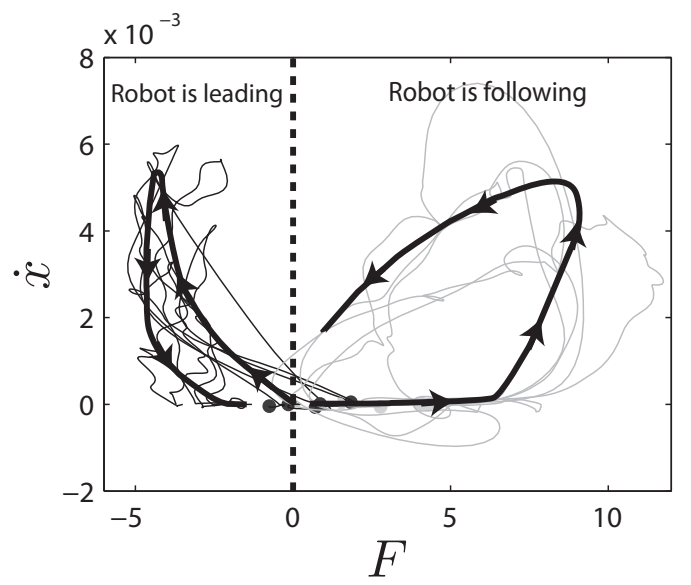

Fig. 2. Resulting patterns in leader and follower teaching demonstration in the force/velocity space.

reproduce the demonstrated task. This framework has been used successfully in 'stand-alone' tasks (see e.g. [13]), and we evaluate its usability in physical collaborative tasks.

The data recorded during the demonstrations of the collaborative lifting task is encoded into a GMM with $K$ components representing the local correlations of the variables in the data-set. The GMM is trained to find the parameters $\lambda=\{\mu, \Sigma\}$ that maximize the likelihood of $P(\xi \mid \lambda)$, where $\xi$ is the data-set. For each component $i, \mu_{i}$ and $\Sigma_{i}$ are the mean vector and covariance matrix of the Gaussian distribution.

The data encoded by the GMM can be reconstructed using a GMR process. If we split the different variables into one output variable $\xi^{\mathcal{O}}$ and a set of input variables $\xi^{\mathcal{I}}$, then for each component $i$ of the GMM, the mean vectors and covariance matrices have the following structure:

$$
\mu_{i}=\left[\begin{array}{l}
\mu_{i}^{\mathcal{I}} \\
\mu_{i}^{\mathcal{O}}
\end{array}\right], \Sigma_{i}=\left[\begin{array}{c}
\Sigma_{i}^{\mathcal{I}} \Sigma_{i}^{\mathcal{I O}} \\
\Sigma_{i}^{\mathcal{O}} \Sigma_{i}^{\mathcal{O}}
\end{array}\right]
$$

The estimate of an output $\hat{\xi^{\mathcal{O}}}$ can be computed by:

$$
\hat{\xi^{\mathcal{O}}}=\sum_{i=1}^{K} h_{i}(\xi)\left(\mu_{i}^{\mathcal{O}}+\Sigma_{i}^{\mathcal{O}}\left(\Sigma_{i}^{\mathcal{I}}\right)^{-1}\left(\xi^{\mathcal{I}}-\mu_{i}^{\mathcal{I}}\right)\right),
$$

where $h_{i}(\xi)$ weights the influence of the Gaussians and is defined by:

$$
h_{i}(\xi)=\frac{\mathcal{N}\left(\xi ; \mu_{i}, \Sigma_{i}\right)}{\sum_{j=1}^{K} \mathcal{N}\left(\xi ; \mu_{j}, \Sigma_{j}\right)}
$$

\section{B. Control scheme}

Using Eq. (2), a target velocity $\hat{\dot{x}}$ will be computed from the actual position $x$ of the gripper and the measured force $F$, and a target position $\hat{x}$ will be computed from the actual velocity $\dot{x}$ of the gripper and $F$. From $\hat{x}$ and $\hat{\dot{x}}$, a reference velocity $\dot{x}^{*}$ is computed for an inner velocity controller of the robot by numerically integrating the following equation:

$$
\ddot{x}^{*}=\kappa^{\mathcal{P}}(\hat{x}-x)+\kappa^{\mathcal{\nu}}(\hat{\dot{x}}-\dot{x})
$$

Eq. 4 has two terms: the position term attracts the robot to the reference position $\hat{x}$ to avoid moving away to an unlearned situation, while the velocity term allows to reproduce the learned dynamics, see more details in [9].

\section{Encoding of the lifting task}

As explained in section II-C, two scenarios have been demonstrated to the robot: one were it behaved as a leader, and one where it behaved as a follower. The resulting dataset have been encoded into two different models, which have been merged into one single model for the reproduction phase. Therefore, the robot can adopt either behavior depending on the user preference. The resulting GMM is depicted on Fig. 3.
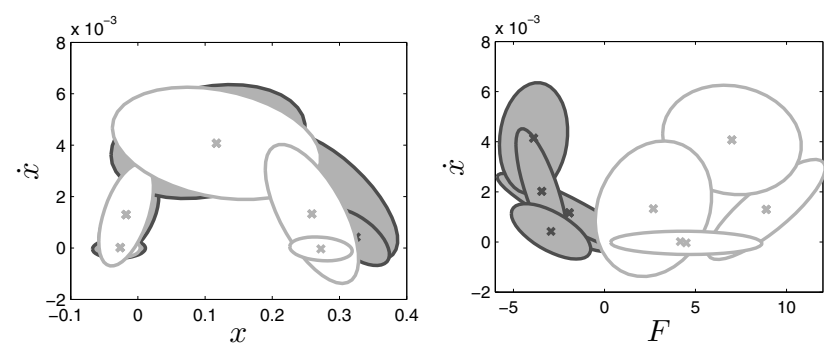

Fig. 3. GMM models encoding the relationships between the velocity and the position, and the velocity and the measured force. The gray components of the left correspond to a leader behavior of the robot, and the mixture on the right (white ellipses) correspond to a follower behavior from the robot.

\section{TASK REPRODUCTION}

This section describes the experimental setup used for the reproductions, and evaluates the usability and robustness of the proposed method. We also show how the experimental results support our behavior switching hypothesis suggested in [7].

\section{A. Reproduction setup}

The setup used for the reproduction of the demonstrated task is the same as during the teaching phase, except that the robot is now acting autonomously, instead of being teleoperated. The velocity of the end-effector of the robot is computed at each control iteration using Eq (4).

Different subjects were asked to lift the object together with the robot. The subject were not given any specific instructions on how they must behave, apart that they had to lift the object naturally together with the robotic partner; therefore, they were not asked to behave as leaders or followers. Figure 4 shows an autonomous replication of a beam lifting task by HRP-2 jointly with a human task-partner (see also the companion video).

\section{B. Experimental results}

1) Successful task reproductions: The reproduction was performed with 13 subjects, not including the two partners involved in the teaching process. 71 lifting task trials have been performed among which 38 were successful replications. In the general case, where the subjects performed 

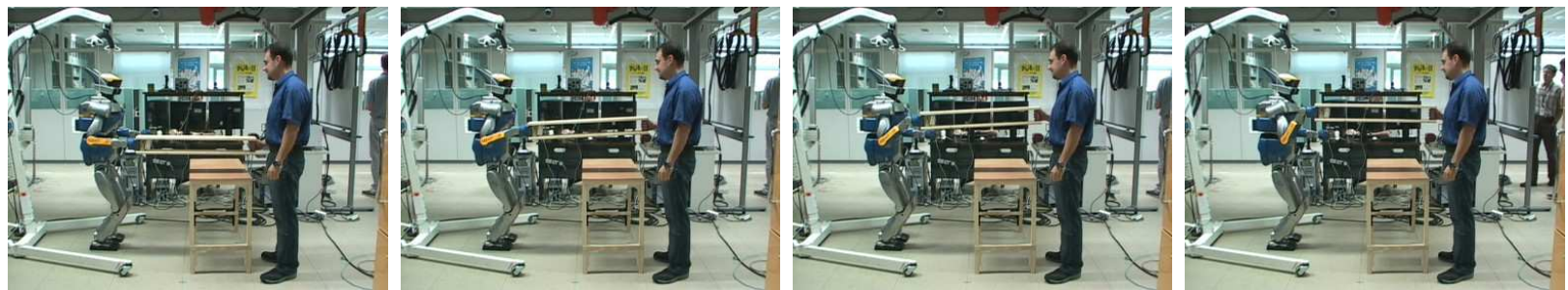

Fig. 4. Autonomous reproduction of a beam lifting task by HRP-2 jointly with a human task-partner.

the task naturally, the robot lifted the object smoothly. As long as the subjects exhibited a behavior close to either of the demonstrated scenarios, the robot behaved properly and the object reached a target position with a smooth velocity profile, even if, as will be seen later, some of the partners switched from one behavior to another.

The about $50 \%$ success rate is not surprising, since the demonstrations were not performed with much variability in the velocity and force profiles. Therefore, the adaptation capabilities of the robot were limited. Extending our model to various velocity and force profiles will be addressed in our future work. This success rate is nevertheless encouraging since it suggests that the approach is quite robust to the variability of human partners.

Given the limited adaptation capabilities of the robot, this success rate can be considered relatively high. This illustrates the challenge in evaluating models and algorithms in haptic collaboration between human operators and robotic systems: during the demonstrations, the robot only interacted with two different human partners. In theory, our model should not have allowed generalization to different human partners. However, in our context, the robot and the human have a symmetric role, and the robot is not the only partner to adapt. It seems that the subjects who participated to the reproduction experiments adapted to the robot controller so as to perform the task successfully.

Most of the 33 failed attempts corresponded to the cases where the object could be lifted, but not with the demonstrated dynamics. However, even when the dynamics of the reproduced motion differed from the demonstrated one, the overall behavior of the system was similar. This is illustrated in Fig. 5, where we see that the dynamics of the motion is similar to the demonstrated one, except that the pattern seems to be stretched along the force dimension, and shifted towards the positive forces. This suggests that it should be possible to extract a general pattern which would be invariant for all partners after rescaling; this can be an interesting issue toward a more robust controller, and will be thoroughly investigated.

2) Reaction to undemonstrated behavior: To test the limits of our current model, we also tried to adopt a very different behavior during some of the trials: at one point in the task, the human subject had to stop abruptly. Due to the nature of the encoded force-velocity relationship, the robot interpreted this as the human having a follower behavior. Indeed, as can be seen in Fig. 2, the Gaussian components

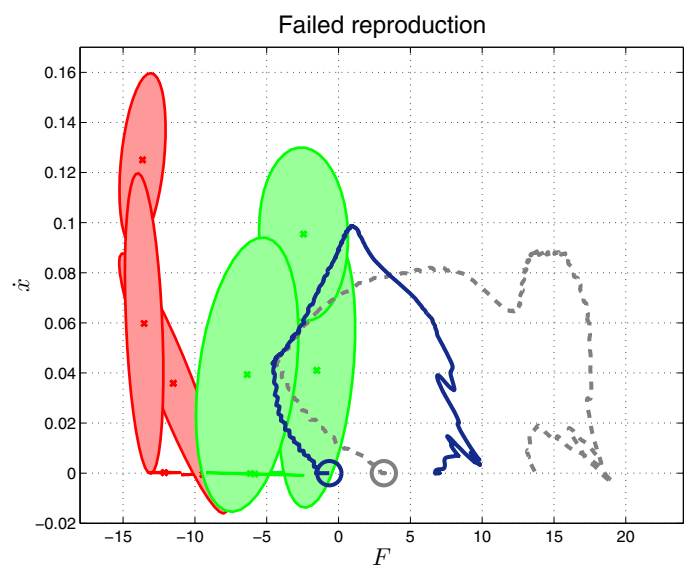

Fig. 5. Failed reproduction attempt. The object is successfully lifted, but the dynamics of the motion differs from the demonstrated one. Notice, however, that the obtained dynamics is similar to the demonstrated one when the robot is behaving as a follower (see Fig. 6).

encoding the data recorded during scenarios where the robot was leading are all located in the subspace of negative forces, which correspond to forces applied downwards by the human operator. In this area of the $F-\dot{x}$ subspace, the velocity and forces are always locally correlated with a negative coefficient, meaning that both variables will vary in opposite directions. As a result, when a human operator tries to brake abruptly before the end of the motion, the GMR process will generate upward reference velocities, while the reference position will follow the trajectory that was usually demonstrated. Hence, the robot resists to the human operator, trying to go even faster upwards as the user is pushing downwards, which is an unstable behavior and leads to jerky motions.

3) Conclusion: The model implemented in this pilot study can be used as a basis for future work as it shows some robustness to conditions that shift from the demonstrated conditions. The experimental results confirm the usability of Programming by Demonstration methodologies to provide robotic systems with the ability to perform collaborative tasks. However, the adaptation capabilities of our current model needs to be improved by additional demonstrating scenarios and task-partners. Finally, the experimental results allow for a qualitative assessment of the proposed method. Additional analysis is also needed to quantitatively evaluate our controllers and compare them to existing techniques in 


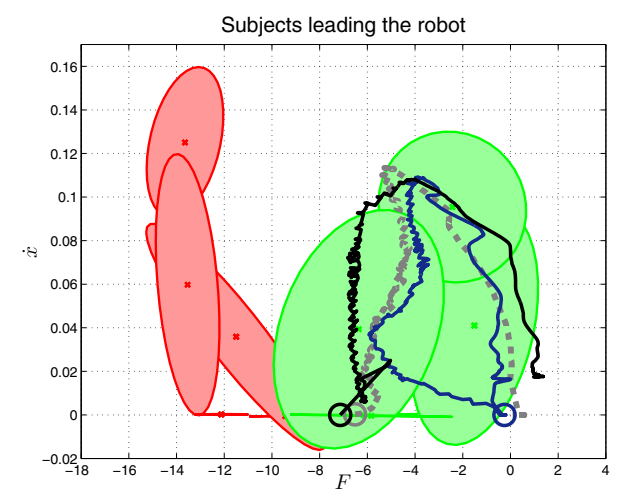

Fig. 6. Human partner behaving closely as a full leader of the lifting task.

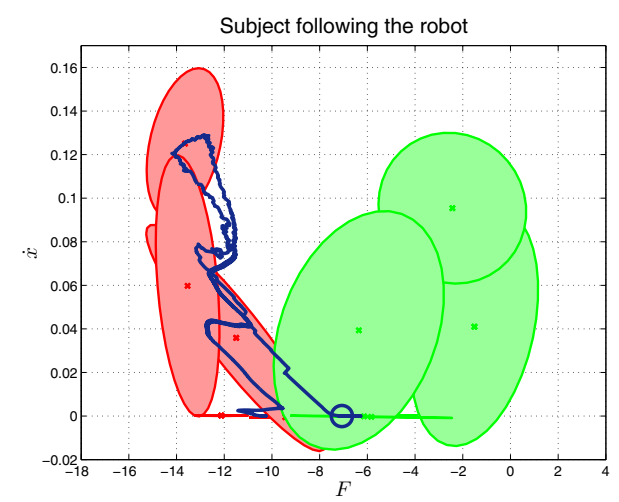

Fig. 7. One human partner behaves closely as a full follower in the lifting task case.

robotics, which is likely to be difficult, given the adaptation capabilities of human subjects.

\section{Preliminary assessment of the leader-follower switching model}

In this paragraph, we will study and comment only the 38 successful trials. We were interested in observing how the subject and the robot would behave if we did not impose any role during the reproduction. Since we use a joint mixture composed from both leader and follower models, we expected that the robot would be able to act either as a leader or as a follower, depending on the preference of the human partner.

The robot behaved as a follower in 23 of the 38 successful trials, as can be seen in the Fig. 6. It is legitimate to conclude that the human partner was leading the robot to the target altitude, since the task was realized. If we confront this results to our dyad physical interaction theory [7], we can only say that the human task-partners' behavior is closer to the leader controller strategy. On the contrary, the Fig. 7 shows a case in which a human partner behaves as a follower.

We were particularily interested to observe switching between the follower and the leader encoded behaviors during the replication of the learned task. In fact, what was taught and what the robot learned is simply to recognize a follower or a leader behavior of the partner and act subsequently in

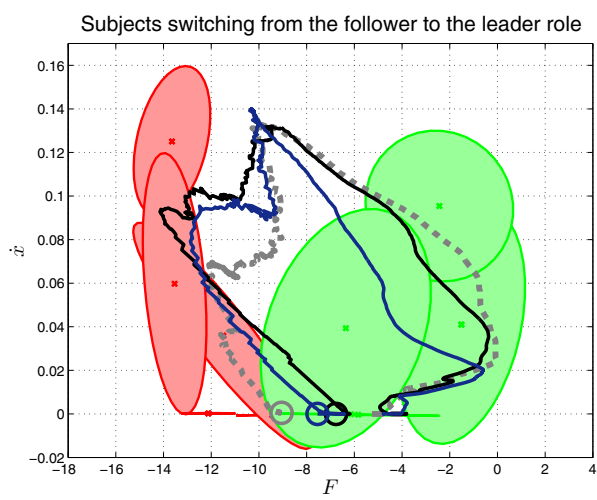

Fig. 8. Human partner behavior switching from a follower to a leader during the lifting task (only three cases are illustrated).

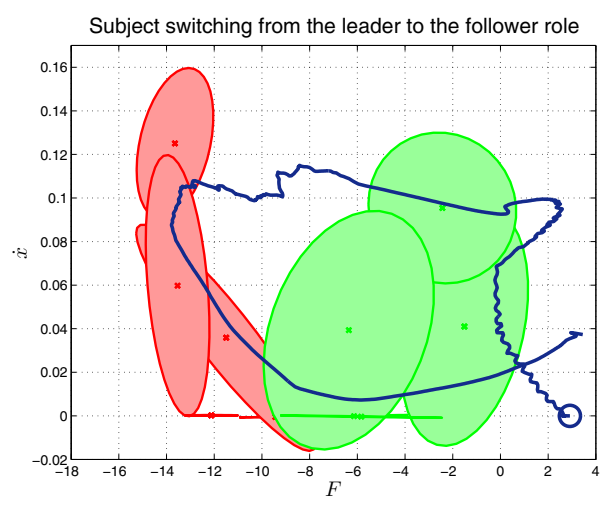

Fig. 9. Illustration of a human partner behavior realizing a double switching of the behavior in a single lifting task.

a complementary way. Note that during the learning phase, see details in [9], we affixed such a role to each partner (teacher and task-partners). It is certainly easy to force the robot to behave strictly as a pure leader or a pure follower; the results show that human operator (task-partners) also did their best to achieve strictly one of the two behaviors. However, when we replicated the learned lifting task on the humanoid robot, we did not instruct the human partners involved in the experiment to behave in either of these two extreme modes. Indeed, we simply asked the human partner to lift the object together with the robot.

Fig. 8 illustrates 3 cases among 14 observed trials where a human partner switched the behavior from a follower to a leader one. Only one subject started as a leader and performed a double switching as can be seen in Fig. 9. What is important for the time being, is that this switching of the behavior seems to agree with our theory and model on dyad interaction [7], [8] in which we claim that the behavior of each dyad is a continuous switch between two extreme theoretical behaviors which are follower or leader, and synchrony is realized through a continuous adjustment of this switch. Here, the humanoid robot recognizes a change of the behavior of the human partner and adapted continuously to that change. 
Note that this switching could not have been obtained if we had used Hidden Markov Models as proposed in [9]. HMMs would have encoded temporal information which would have resulted in very low switching probabilities from the components of the mixture representing the leader behavior to those representing the follower behavior, and vice-versa.

\section{CONCLUSIONS AND FUTURE WORKS}

This paper deals with one aspect of Human-Robot interactions, which aims at endowing a robotic platform with the ability to perform collaborative tasks. We proposed to use a probabilistic framework based on Gaussian Mixture Models and Gaussian Mixture Regression to encode the local correlations between different variables that characterize collaborative tasks and use the encoded data to reproduce demonstrated tasks. We aim at investigating whether this learning technique can encapsulate all the complexity of collaborative tasks, including the dynamics of the motions and the communication process that takes place between the partners to negotiate a common control strategy. In this paper, we focused on the case of one dimensional collaborative lifting task, with two sets of stereotypical demonstrated scenarios, corresponding to two different role distributions among the human and robotic partners. We investigated whether the proposed probabilistic framework allows the learning and reproduction of this simplified scenario. The first experimental results are promising: almost all subjects could perform the lifting task smoothly, and the model showed some robustness to behaviors that were not demonstrated. This generalization capability is limited though, and deeper investigations are needed to extend the model so that more complex situations can be addressed with the proposed method. Future work will focus on quality assessment of the proposed controller, extension to more complex 3D scenarios, improvement of the overall task performance, and assessment of the model of dyad interaction based on homotopy switching.

\section{ACKNOWLEDGMENTS}

This work is partially supported by grants from: the FETPresence under FP6 IP ImmerSence, Contract No. 27141 www.immersence.info and the FP6 ROBOT@CWE, Contract No. 34002 www. robot-at-cwe.eu.

\section{REFERENCES}

[1] N. Hogan, "Impedance Control: An Approach to Manipulation, Part 1 - Theory," Journal of Dynamic Systems, Measurement, and Control, vol. 107, pp. 1-7, March 1985.

[2] T. Tsumugiwa, R. Yokogawa, and K. Hara, "Variable impedance control with regard to working process for man-machine cooperationwork system," in IEEE/RSJ Int. Conf. on Robots and Intelligent Systems, Maui, Hawaii, October-November 2001, pp. 1564-1569.

[3] Y. Maeda, T. Hara, and T. Arai, "Human-robot cooperative manipulation with motion estimation," in IEEE/RSJ Int. Conf. on Robots and Intelligent Systems, Maui, Hawaii, October-November 2001, pp. 2240-2245.

[4] M. Rahman, R. Ikeura, and K. Mizutani, "Investigation of the impedance characteristic of human arm for development of robots to cooperate with humans," JSME Int. Journal Series C, Mechanical Systems, Machine Elements and Manufacturing, vol. 45, no. 2, pp. 510-518, 2002.
[5] V. Duchaine and C. M. Gosselin, "General model of human-robot cooperation using a novel velocity based variable impedance control," in Joint EuroHaptics Conference and Symposium on Haptic Interfaces for Virtual Environment and Teleoperator Systems. Washington, DC, USA: IEEE Computer Society, 2007, pp. 446-451.

[6] B. Corteville, E. Aertbelien, H. Bruyninckx, J. D. Schutter, and H. V. Brussel, "Human-inspired robot assistant for fast point-to-point movements." in IEEE Int. Conf. on Robotics and Automation. IEEE, 2007, pp. 3639-3644.

[7] P. Evrard and A. Kheddar, "Homotopy switching model for dyad haptic interaction in physical collaborative tasks," in Joint EuroHaptics Conference and Symposium on Haptic Interfaces for Virtual Environment and Teleoperator Systems, Salt Lake City, USA, March 2009.

[8] —_ "Homotopy-based controller for human-robot interaction," in 18th IEEE Int. Symposium on Robot and Human Interactive Communication (Ro-Man 2009), Toyama, Japan, 27 september -2 october 2009.

[9] S. Calinon, P. Evrard, E. Gribovskaya, A. Billard, and A. Kheddar, "Learning collaborative manipulation tasks by demonstration using a haptic interface," in IEEE Int. Conf. on Advanced Robotics, Munich, Germany, June 2009.

[10] S. Miossec and A. Kheddar, "Human hand motion in cooperative tasks: moving object case study," in IEEE Int. Conf. on Robotics and Biomimetics, Bangkok, Thailand, December 14-17 2008.

[11] A. Kheddar, V. Gourishankar, and P. Evrard, "A phantom device with 6dof force feedback and sensing capabilities." in EuroHaptics, ser. Lecture Notes in Computer Science, M. Ferre, Ed., vol. 5024. Springer, 2008, pp. 146-150. [Online]. Available: http://dblp.uni-trier. $\mathrm{de} / \mathrm{db} /$ conf/haptics/eurohaptics2008.html $\backslash$ \#KheddarGE08

[12] A. Billard, S. Calinon, R. Dillmann, and S. Schaal, "Robot Programming by Demonstration," in Handbook of Robotics. MIT Press, 2008, vol. chapter 59 .

[13] S. Calinon, F. Guenter, and A. Billard, "On learning, representing and generalizing a task in a humanoid robot," IEEE Transactions on Systems, Man and Cybernetics, Part B. Special issue on robot learning by observation, demonstration and imitation, vol. 37 , no. 2, pp. 286298, 2007. 